\title{
Coordenadas geográficas na estimativa das temperaturas máxima e média decendiais do ar no Estado do Rio Grande do Sul
}

\author{
Geographic coordinates in the ten-day maximum and mean air temperature estimation in the State of \\ Rio Grande do Sul, Brazil
}

\begin{abstract}
Alberto Cargnelutti Filho ${ }^{\mathrm{I}}$ Jaime Ricardo Tavares Maluf ${ }^{\mathrm{II}}$ Ronaldo Matzenauer ${ }^{\mathrm{II}}$
\end{abstract}
\section{RESUMO}

A partir dos dados referentes à temperatura máxima média decendial (Tx) e à temperatura média decendial (Tm) do ar de 41 municípios do Estado do Rio Grande do Sul, de 1945 a 1974, este trabalho teve como objetivo verificar se a Tx e a Tm podem ser estimadas em função da altitude, latitude e longitude. Para cada um dos 36 decêndios do ano, realizouse análise de correlação e estimaram-se os parâmetros do modelo das equações de regressão linear múltipla, considerando Tx e Tm como variável dependente e altitude, latitude e longitude como variáveis independentes. Na validação dos modelos de estimativa da Tx e Tm, usou-se o coeficiente de correlação linear de Pearson, entre a Tx e a Tm estimada e a Tx e a Tm observada em dez municípios do Estado, com dados da série de observações meteorológicas de 1975 a 2004. A temperatura máxima média decendial e a temperatura média decendial podem ser estimadas por meio da altitude, latitude e longitude, em qualquer local e decêndio, no Estado do Rio Grande do Sul.

Palavras-chave: modelagem, matriz matemática, regressão linear múltipla, análise de correlação.

\section{ABSTRACT}

The objective of this research was to estimate tenday maximum (Tx) and mean (Tm) air temperature using altitude and the geographic coordinates latitude and longitude for the Rio Grande do Sul State, Brazil. Normal ten-day maximum and mean air temperature of 41 counties in the State of Rio Grande do Sul, from 1945 to 1974 were used. Correlation analysis and parameters estimate of multiple linear regression equations were performed using $\mathrm{Tx}$ and $\mathrm{Tm}$ as dependent variable and altitude, latitude and longitude as independent variables, for the 36 ten-day periods of the year. Pearson's linear correlation coefficient between estimated and observed Tx and Tm, calculated for tem counties using data of were used as independent data sets. The ten-day maximum and mean air temperature may be estimated from the altitude and the geographic coordinates latitude and longitude in the State of Rio Grande do Sul.

Key words: modeling, mathematics matrix, multiple linear regression, analysis of correlation.

\section{INTRODUÇÃO}

O estudo da disponibilidade térmica do ambiente é realizado com a temperatura do ar em suas diversas modalidades (médias, máximas e mínimas) e escalas (anual, mensal, quinzenal, decendial, diária e horária). Esse estudo é importante para zoneamento de culturas agrícolas, indicação de épocas de semeadura, irrigação, determinação de potencial de produtividade, zoneamento de risco climático, crédito e seguro agrícola.

A temperatura do ar é medida em estações meteorológicas. No entanto, essas estações nem sempre são em número suficiente e com distribuição geográfica satisfatória. Vários trabalhos têm buscado métodos de estimativa de parâmetros meteorológicos, principalmente temperatura, em locais ou regiões carentes de medição. Enfoque amplo tem sido dado à estimativa de temperatura em escala anual e mensal em função das coordenadas geográficas.

A altitude e a latitude foram usadas como variáveis independentes para o estabelecimento de equações de estimativa da temperatura média mensal para o Estado de São Paulo (PINTO et al., 1972), da

IDepartamento de Estatística, Universidade Federal do Rio Grande do Sul (UFRGS). Av. Bento Gonçalves, 9500, Bairro Agronomia,

91509-900. Porto Alegre, RS, Brasil. E-mail: cargnelutti@ufrgs.br. Autor para correspondência.

IIFundação Estadual de Pesquisa Agropecuária (FEPAGRO). Porto Alegre, RS, Brasil. 
temperatura média, máxima e mínima mensal no Estado do Paraná (PINTO \& ALFONSI, 1974), das normais de temperatura média mensal e anual no Estado de Goiás (ALFONSI et al., 1974), da temperatura média da máxima e da mínima mensal no Estado de São Paulo (PEDRO JÚNIOR et al., 1991), da temperatura média mensal e anual do Estado do Rio Grande do Sul (FERREIRA et al., 1971) e de Santa Catarina (FERREIRA et al., 1974), da média da temperatura máxima mensal e anual do Estado do Rio Grande do Sul (BURIOL et al., 1973) e de Santa Catarina (BURIOL et al., 1974), da média da temperatura mínima mensal e anual do Estado do Rio Grande do Sul (ESTEFANEL et al., 1973). De maneira geral, esses estudos revelaram elevados coeficientes de correlação e determinação, comprovando um bom ajuste dos modelos.

Em relação às escala mensal e anual, a estimativa da temperatura do ar mínima, média e máxima, a partir das coordenadas geográficas, tem sido estudada nos Estados de Minas Gerais (COELHO et al., 1973; SEDIYAMA\& MELO JÚNIOR, 1998), do Espírito Santo (FEITOZA et al., 1980a, 1980b), do Piauí (LIMA \& RIBEIRO, 1998), de São Paulo (VALERIANO \& PICINI, 2000) e em regiões do Brasil (OLIVEIRA NETO et al., 2002; MEDEIROS et al., 2005).

A ausência de dados meteorológicos referentes a algumas regiões, associada à facilidade de obtenção de coordenadas geográficas e à importância da temperatura em atividades da agricultura, justifica a elaboração de modelos para estimação de temperatura a partir das coordenadas geográficas. Estimativas em escala decendial possibilitam estudos mais detalhados em relação às escalas mensal e anual. Nesse contexto, CARGNELUTTI FILHO et al. (2006) estabeleceram equações para a estimativa da temperatura mínima média decendial do ar para o Estado do Rio Grande do Sul em função das coordenadas geográficas. No entanto, modelos relacionando as coordenadas geográficas com a temperatura máxima e média, em escala decendial, não foram encontrados para o Estado do Rio Grande do Sul.

Portanto, o objetivo deste trabalho foi verificar, com os dados de 41 municípios do Estado do Rio Grande do Sul, se a temperatura máxima média decendial e a temperatura média decendial do ar podem ser estimadas em função da altitude, latitude e longitude.

\section{MATERIAL E MÉTODOS}

Foram usados dados de temperatura máxima e média do Banco de Dados do Centro de Meteorologia Aplicada, da Fundação Estadual de Pesquisa
Agropecuária e do $8^{\circ}$ Distrito de Meteorologia do Instituto Nacional de Meteorologia de municípios de 41 estações meteorológicas localizadas no Estado do Rio Grande do Sul. As informações foram obtidas em Instituto de Pesquisas Agronômicas (1989) e são referentes ao período com disponibilidade de dados (entre 1945 e 1974) (Tabela 1).

Em cada município, a partir dos dados diários de temperatura máxima, medida em abrigo meteorológico situado a $1,5 \mathrm{~m}$ de altura em relação à superfície do solo, foi calculada a temperatura máxima média decendial e a média da temperatura máxima média decendial (Tx) do período de 1945 a 1974, totalizando 30 anos de observações, nos 36 decêndios do ano (1ำ decêndio de janeiro até 3 o decêndio de dezembro). Este procedimento também foi realizado em relação à temperatura média, obtendo-se a média da temperatura média decendial (Tm) do período de 1945 a 1974.

Os dados de Tx e as coordenadas geográficas altitude (em metros), latitude (em graus valores positivos) e longitude (em graus - valores positivos) dos 41 municípios formaram 36 matrizes (36 decêndios) de $41 \times 4$ (41 observações e 4 variáveis). Trinta e seis matrizes também foram formadas para os dados de Tm e as coordenadas geográficas.

Em cada uma das matrizes (72 matrizes), estimaram-se os coeficientes de correlação linear de Pearson entre as variáveis Tx versus altitude, latitude e longitude, e Tm versus altitude, latitude e longitude e verificou-se a significância pelo teste de $t$ de Student a $5 \%$ de probabilidade.

Em cada matriz foi usado o modelo de regressão linear múltipla com $\mathrm{k}$ variáveis independentes:

$$
Y_{j}=\beta_{0}+\sum_{i=1}^{k} \beta_{i} X_{i j}+\varepsilon_{j} \text {, em que } Y_{j} \text { é o valor }
$$

observado da Tx ou Tm; $\beta_{0}$ é o coeficiente linear; $\beta_{\mathrm{i}}$ é o coeficiente de regressão das variáveis independentes, $\mathrm{X}_{\mathrm{i}} ; \mathrm{X}_{\mathrm{ij}}$ são as variáveis independentes $\mathrm{X}_{\mathrm{i}}$ na observação $\mathrm{j}$; e $\varepsilon_{\mathrm{j}}$ é o erro associado à variável Y na observação $\mathrm{j}$. Nesse modelo, a variável Y é função linear das variáveis independentes, os valores das variáveis independentes são fixos e os erros têm média zero, são homocedásticos, independentes e de distribuição normal (DRAPER \& SMITH, 1996; NETER et al., 1997; HOFFMANN \& VIEIRA, 1998; SOUZA, 1998).

Os parâmetros do modelo das equações de regressão linear múltipla foram estimados considerando Tx e Tm, respectivamente, como variável dependente e as demais [altitude (em metros), latitude (em graus - valores positivos) e longitude (em graus valores positivos)], foram consideradas como independentes.

Em cada decêndio, verificou-se o ajuste dos modelos de estimativa da $\mathrm{Tx}$, pelo coeficiente de 
Tabela 1 - Coordenadas geográficas das estações meteorológicas localizadas em municípios do Estado do Rio Grande do Sul.

\begin{tabular}{|c|c|c|c|c|c|c|c|}
\hline \multirow{3}{*}{$\begin{array}{l}\text { Município } \\
\text { Alegrete }\end{array}$} & \multirow{3}{*}{$\begin{array}{c}\text { Altitude (m) } \\
121,00\end{array}$} & \multirow{2}{*}{\multicolumn{3}{|c|}{--------Latitude (S)--------- }} & \multirow{2}{*}{\multicolumn{3}{|c|}{--------Longitude (W)--------- }} \\
\hline & & & & & & & \\
\hline & & $29^{\circ}$ & $41^{\prime}$ & $47 ”$ & $55^{\circ}$ & 31 & $15 "$ \\
\hline Bagé & 242,31 & $31^{\circ}$ & $20{ }^{\prime}$ & $13 ”$ & $54^{\circ}$ & $06^{\prime}$ & $21 "$ \\
\hline Bento Gonçalves & 640,00 & $29^{\circ}$ & $09^{\prime}$ & $00 ”$ & $51^{\circ}$ & 31 & $00 "$ \\
\hline Bom Jesus & 1047,50 & $28^{\circ}$ & $40^{\prime}$ & $10 ”$ & $50^{\circ}$ & $26^{\prime}$ & $25 "$ \\
\hline Caçapava do Sul & 450,00 & $30^{\circ}$ & 30 & $32 "$ & $53^{\circ}$ & 29 & $22 "$ \\
\hline Cachoeira do Sul & 72,71 & $30^{\circ}$ & $02^{\prime}$ & $45 ”$ & $52^{\circ}$ & $53^{\prime}$ & $39 ”$ \\
\hline Caxias do Sul & 759,60 & $29^{\circ}$ & $10{ }^{\prime}$ & $25 ”$ & $51^{\circ}$ & $12^{\prime}$ & $21 "$ \\
\hline Cruz Alta & 472,50 & $28^{\circ}$ & $38^{\prime}$ & $21 ”$ & $53^{\circ}$ & $36^{\prime}$ & $34 "$ \\
\hline Dom Pedrito & 140,00 & $30^{\circ}$ & 58 & $57 ”$ & $54^{\circ}$ & $39^{\prime}$ & $56 "$ \\
\hline Guaporé & 471,51 & $28^{\circ}$ & $55^{\prime}$ & $44 ”$ & $51^{\circ}$ & $54 '$ & $45^{\prime \prime}$ \\
\hline Irai & 247,10 & $27^{\circ}$ & $11^{\prime}$ & $45 ”$ & $53^{\circ}$ & $14^{\prime}$ & $01 "$ \\
\hline Itaqui & 76,00 & $29^{\circ}$ & $07^{\prime}$ & $10 "$ & $56^{\circ}$ & $32^{\prime}$ & $52 ”$ \\
\hline Jaguarão & 50,00 & $32^{\circ}$ & $33^{\prime}$ & $32 ”$ & $53^{\circ}$ & $23^{\prime}$ & $20 "$ \\
\hline Lagoa Vermelha & 840,00 & $28^{\circ}$ & $13^{\prime}$ & $35 ”$ & $51^{\circ}$ & $30^{\prime}$ & $51 ”$ \\
\hline Marcelino Ramos & 414,17 & $27^{\circ}$ & $27^{\prime}$ & $40 ”$ & $51^{\circ}$ & $54^{\prime}$ & $22 ”$ \\
\hline Mostardas & 3,00 & $31^{\circ}$ & $06^{\prime}$ & $25 "$ & $50^{\circ}$ & $55^{\prime}$ & $16 "$ \\
\hline Palmeira das Missões & 634,00 & $27^{\circ}$ & $53^{\prime}$ & $55^{\prime \prime}$ & $53^{\circ}$ & $26^{\prime}$ & $45^{\prime \prime}$ \\
\hline Passo Fundo & 684,05 & $28^{\circ}$ & $13^{\prime}$ & $39 ”$ & $52^{\circ}$ & 24 & $33 ”$ \\
\hline Pelotas & 13,00 & $31^{\circ}$ & $47^{\prime}$ & $00 ”$ & $52^{\circ}$ & $25^{\prime}$ & $00 ”$ \\
\hline Piratini & 345,00 & $31^{\circ}$ & $26^{\prime}$ & $54 ”$ & $53^{\circ}$ & $06^{\prime}$ & $09 ”$ \\
\hline Porto Alegre & 10,00 & $30^{\circ}$ & $01^{\prime}$ & $53 ”$ & $51^{\circ}$ & 13 & $19 "$ \\
\hline Rio Grande & 2,46 & $32^{\circ}$ & $01^{\prime}$ & $44 "$ & $52^{\circ}$ & 05 & $40 ”$ \\
\hline Santa Cruz do Sul & 52,51 & $29^{\circ}$ & $43^{\prime}$ & $05 ”$ & $52^{\circ}$ & $25^{\prime}$ & $45^{\prime \prime}$ \\
\hline Santa Maria & 95,00 & $29^{\circ}$ & $41^{\prime}$ & $25 "$ & $53^{\circ}$ & $42^{\prime}$ & $42 "$ \\
\hline Santa Rosa & 360,00 & $27^{\circ}$ & $51^{\prime}$ & $50 ”$ & $54^{\circ}$ & $25^{\prime}$ & $59 ”$ \\
\hline Santa Vitória do Palmar & 24,01 & $33^{\circ}$ & $31^{\prime}$ & $14 "$ & $53^{\circ}$ & $21^{\prime}$ & $47 "$ \\
\hline Santana do Livramento & 328,00 & $30^{\circ}$ & $50^{\prime}$ & $18 ”$ & $55^{\circ}$ & $36^{\prime}$ & $56 "$ \\
\hline Santiago & 439,00 & $29^{\circ}$ & $11^{\prime}$ & $00 ”$ & $54^{\circ}$ & $53^{\prime}$ & $10 ”$ \\
\hline Santo Ângelo & 284,50 & $28^{\circ}$ & $18^{\prime}$ & $14 "$ & $54^{\circ}$ & 15 & $52 ”$ \\
\hline São Borja & 96,00 & $28^{\circ}$ & $39^{\prime}$ & $44 "$ & $56^{\circ}$ & $00^{\prime}$ & $15^{\prime \prime}$ \\
\hline São Francisco de Paula & 922,00 & $29^{\circ}$ & 20 & $00 ”$ & $50^{\circ}$ & 31 & $21 ”$ \\
\hline São Gabriel & 124,00 & $30^{\circ}$ & $20^{\prime}$ & $27 "$ & $54^{\circ}$ & $19^{\prime}$ & $01 ”$ \\
\hline São Luiz Gonzaga & 245,11 & $28^{\circ}$ & $24^{\prime}$ & $27 ”$ & $55^{\circ}$ & $01^{\prime}$ & $18 ”$ \\
\hline Soledade & 720,00 & $28^{\circ}$ & $500^{\prime}$ & $14 "$ & $52^{\circ}$ & $31^{\prime}$ & $00 ”$ \\
\hline Tapes & 5,00 & $30^{\circ}$ & 50 & $00 ”$ & $51^{\circ}$ & $35^{\prime}$ & $00 ”$ \\
\hline Taquara & 35,00 & $29^{\circ}$ & $45^{\prime}$ & $00 ”$ & $50^{\circ}$ & $45^{\prime}$ & $00 "$ \\
\hline Taquarí & 76,00 & $29^{\circ}$ & $48^{\prime}$ & $15 "$ & $51^{\circ}$ & $49^{\prime}$ & $30 "$ \\
\hline Torres & 4,66 & $29^{\circ}$ & $21^{\prime}$ & $34 "$ & $49^{\circ}$ & $43^{\prime}$ & $39 ”$ \\
\hline Uruguaiana & 62,31 & $29^{\circ}$ & $45^{\prime}$ & $23 ”$ & $57^{\circ}$ & $05^{\prime}$ & $12 ”$ \\
\hline Vacaria & 954,60 & $28^{\circ}$ & $33^{\prime}$ & $00 ”$ & $50^{\circ}$ & $42^{\prime}$ & $21 "$ \\
\hline Viamão & 48,77 & $30^{\circ}$ & $05^{\prime}$ & $00 ”$ & $50^{\circ}$ & $47^{\prime}$ & $00 "$ \\
\hline
\end{tabular}

${ }^{(1)}$ Fonte: Instituto de Pesquisas Agronômicas (1989).

correlação linear de Pearson entre a Tx estimada pelo modelo e a Tx observada. A significância foi verificada pelo teste de $t$ de Student a $1 \%$ de probabilidade. Este mesmo procedimento foi realizado em relação à $\mathrm{Tm}$. Para isso, foram usados dados de dez municípios (Encruzilhada do Sul, Júlio de Castilhos, Rio Grande,
Santa Maria, Santa Rosa, São Borja, São Gabriel, Taquari, Vacaria e Veranópolis) do Estado do Rio Grande do Sul, de 1975 a 2004, com alguns municípios diferentes daqueles utilizados para obtenção das estimativas dos parâmetros do modelo. As análises foram realizadas com o auxílio do programa GENES (CRUZ, 2001) e do aplicativo MSOffice Excel.

Ciência Rural, v.38, n.9, dez, 2008. 


\section{RESULTADOS E DISCUSSÃO}

A magnitude e o sinal do coeficiente de correlação linear de Pearson, da variável temperatura máxima média decendial (Tx) do ar e da temperatura média decendial (Tm) do ar com as variáveis altitude, latitude e longitude foram diferentes (Tabela 2). Nos casos em que houve correlação $(\mathrm{P} \leq 0,05)$, esta foi negativa em relação à altitude e latitude e positiva em relação à longitude. Resultados semelhantes em relação à estimativa da temperatura mínima média decendial (Tmin) do ar foram encontrados por CARGNELUTTI FILHO et al. (2006).

Houve correlação negativa e significativa $(\mathrm{P} \leq 0,05)$ entre temperatura máxima média decendial $(\mathrm{Tx})$ e altitude, em 27 decêndios (75\%), e entre temperatura média decendial $(\mathrm{Tm})$ e altitude em todos decêndios, evidenciando diminuição da $\mathrm{Tx}$ e da Tm com o acréscimo da altitude (Tabela 2). Resultado esperado e de acordo o gradiente térmico vertical na troposfera. De maneira geral, a magnitude dos coeficientes de correlação da Tx e da altitude foi menor do que os obtidos por CARGNELUTTI FILHO et al. (2006) entre a temperatura mínima média decendial (Tmin) do ar e a altitude. Em relação à Tm e altitude, a magnitude dos coeficientes foi superior à Tx e altitude e semelhante à Tmin e altitude (CARGNELUTTI FILHO et al., 2006). Esses resultados sugerem maior influência da altitude na estimativa da $\mathrm{Tm}$ em relação à estimativa de $\mathrm{Tx}$. CARGNELUTTI FILHO et al. (2006) constataram que a altitude e a latitude, nessa ordem, exercem maior efeito sobre a Tmin no Estado do Rio Grande do Sul.

$O$ coeficiente de correlação entre Tx e latitude foi negativo e significativo $(\mathrm{P} \leq 0,05)$ nos decêndios dos meses de junho a novembro (18 decêndios) e entre Tm e latitude no segundo e no terceiro decêndios de agosto e no primeiro e no segundo de setembro. Nos demais decêndios não houve relação linear entre essas variáveis (Tabela 2). CARGNELUTTI FILHO et al. (2006) relatam que o coeficiente de correlação entre a Tmin e a latitude foi positivo ou negativo, de baixa magnitude e não-significativo em todos os decêndios. Esses autores destacam também que a presença da latitude nos modelos de estimativa é explicada pelo efeito indireto positivo e de alta magnitude via altitude sobre a Tmin, que impede a manifestação dessa correlação, e também em virtude do efeito direto da latitude sobre a Tmin.

Em relação à $T x$ e longitude, houve correlação positiva e significativa $(\mathrm{P} \leq 0,05)$ em trinta decêndios $(83,33 \%)$ e os demais coeficientes foram positivos de baixa magnitude e não houve significância da associação linear. Entre Tm e longitude houve associação linear positiva e significativa em 27 decêndios (75,00\%). Esses resultados sugerem maior efeito da longitude na estimativa de Tx e Tm, quando comparada à estimativa de Tmin (CARGNELUTTI FILHO et al., 2006), que encontrou correlação em apenas oito decêndios $(22,22 \%)$, sendo que nos demais não houve significância da associação linear. Justifica-se assim a presença da longitude nos modelos de estimativa de Tx e Tm (Tabelas 3 e 4).

$O$ coeficiente de determinação $\left(R^{2}\right)$ da equação de regressão linear múltipla da Tx em função da altitude, latitude e longitude oscilou de $71,11 \%$ ( $2^{\circ}$ decêndio de agosto) a $89,12 \%$ (2o decêndio de julho) com média de 79,85\%. Esses resultados evidenciam que, no 2 decêndio de agosto, $71,11 \%$ da variação da Tx é explicada pelas variáveis que constam no modelo (altitude, latitude e longitude) e outras variáveis não incluídas no modelo (por exemplo, a distância do local ao oceano) explicam o restante da variação $(28,89 \%)$. No $2^{\circ}$ decêndio de julho, $89,12 \%$ da variação da Tx é explicada pelas variáveis incluídas no modelo e 10,88\% por outras variáveis (Tabela 3 ). Em relação à $T m, o R^{2}$ oscilou de 75,17\% ( $1^{\circ}$ decêndio de agosto) a 93,06\% ( $2^{\circ}$ decêndio de fevereiro) com média de 86,05\% (Tabela 4). Então, infere-se bom ajuste dos modelos para estimativa da Tx e Tm usando a altitude, latitude e longitude em todos os decêndios no Estado do Rio Grande do Sul.

Nos modelos de estimativa de Tx, há, em geral, maiores valores de $\mathrm{R}^{2}$ (melhores ajustes do modelo) nos meses centrais do ano com leve redução em direção aos primeiros e últimos meses do ano. Em relação à Tm, ocorreu comportamento inverso, ou seja, os primeiros e os últimos meses do ano apresentaram os maiores valores de $\mathrm{R}^{2}$, com diminuição gradativa em direção aos meses centrais do ano. Valores menores de coeficientes de determinação para os meses de inverno (meses centrais do ano) são relatados por PINTO \& ALFONSI (1974)e CARGNELUTTIFILHO et al. (2006). Quanto à magnitude de $\mathrm{R}^{2}$, de maneira geral, maiores estimativas foram obtidas em relação à Tm (média de $\mathrm{R}^{2}=86,05 \%$ ), Tx (média de $\mathrm{R}^{2}=79,85 \%$ ) e Tmin (média de $\mathrm{R}^{2}=66,49 \%$ ) (CARGNELUTTI FILHO et al., 2006), evidenciando maior e menor precisão, respectivamente, na estimativa da temperatura do ar.

Em relação à $\mathrm{Tx}$, a estimativa do parâmetro relacionado à altitude $\left(\beta_{1}\right)$ nos 36 decêndios do ano oscilou entre $-0,00560095$ ( 2 o decêndio de abril) e 0,00319888 ( 1 - decêndio de setembro) com média de 0,00454240 (Tabela 3 ), já a Tm variou de $-0,00548227$ ( $2^{\circ}$ decêndio de março) a $-0,00341846$ ( 2 - decêndio de agosto) com média de -0,00459012 (Tabela 4). Esses resultados indicam que, em média, ocorre decréscimo 
Tabela 2 - Estimativa dos coeficientes de correlação linear de Pearson, da temperatura máxima média decendial (Tx) e da temperatura média decendial (Tm) com a altitude, a latitude e a longitude, no Estado do Rio Grande do Sul, com dados de 1945 a 1974.

\begin{tabular}{|c|c|c|c|c|c|c|c|}
\hline \multirow{2}{*}{ Mês } & \multirow{2}{*}{ Decêndio } & \multicolumn{3}{|c|}{---------------------Tx com-------------------- } & \multicolumn{3}{|c|}{-------------------Tm com------------------- } \\
\hline & & Altitude & Latitude & Longitude & Altitude & Latitude & Longitude \\
\hline \multirow{3}{*}{ janeiro } & 1 & $-0,5271^{*}$ & $-0,1609^{\mathrm{ns}}$ & $0,6551^{*}$ & $-0,7088^{*}$ & $-0,0382^{\mathrm{ns}}$ & $0,6643^{*}$ \\
\hline & 2 & $-0,5485^{*}$ & $-0,1582^{\mathrm{ns}}$ & $0,6747 *$ & $-0,7790 *$ & $0,0314^{\mathrm{ns}}$ & $0,6345^{*}$ \\
\hline & 3 & $-0,5683^{*}$ & $-0,1244^{\mathrm{ns}}$ & $0,6790 *$ & $-0,7619^{*}$ & $0,0343^{\mathrm{ns}}$ & $0,6550^{*}$ \\
\hline \multirow{3}{*}{ fevereiro } & 1 & $-0,5407 *$ & $-0,2091^{\mathrm{ns}}$ & $0,6592 *$ & $-0,7609 *$ & $-0,0193^{\text {ns }}$ & $0,5916^{*}$ \\
\hline & 2 & $-0,6294 *$ & $-0,0888^{\mathrm{ns}}$ & $0,6828 *$ & $-0,8295^{*}$ & $0,0802^{\mathrm{ns}}$ & $0,5646^{*}$ \\
\hline & 3 & $-0,5976^{*}$ & $-0,1287^{\mathrm{ns}}$ & $0,6652 *$ & $-0,8140^{*}$ & $0,0787^{\mathrm{ns}}$ & $0,5869^{*}$ \\
\hline \multirow{3}{*}{ março } & 1 & $-0,5817^{*}$ & $-0,1921^{\mathrm{ns}}$ & $0,5959^{*}$ & $-0,8301^{*}$ & $0,0993^{\mathrm{ns}}$ & $0,5295^{*}$ \\
\hline & 2 & $-0,6449^{*}$ & $-0,1448^{\mathrm{ns}}$ & $0,5205^{*}$ & $-0,8365^{*}$ & $0,0504^{\mathrm{ns}}$ & $0,4275^{*}$ \\
\hline & 3 & $-0,6393^{*}$ & $-0,1400^{\mathrm{ns}}$ & $0,5381^{*}$ & $-0,8407^{*}$ & $0,0730^{\mathrm{ns}}$ & $0,4574 *$ \\
\hline \multirow{3}{*}{ abril } & 1 & $-0,6469^{*}$ & $-0,1968^{\mathrm{ns}}$ & $0,5075^{*}$ & $-0,8253^{*}$ & $0,0643^{\mathrm{ns}}$ & $0,3761 *$ \\
\hline & 2 & $-0,6585^{*}$ & $-0,2005^{\mathrm{ns}}$ & $0,4346^{*}$ & $-0,8178^{*}$ & $0,0333^{\mathrm{ns}}$ & $0,3210^{*}$ \\
\hline & 3 & $-0,6478^{*}$ & $-0,2065^{\mathrm{ns}}$ & $0,3470^{*}$ & $-0,8345^{*}$ & $0,0566^{\mathrm{ns}}$ & $0,2868^{\mathrm{ns}}$ \\
\hline \multirow{3}{*}{ maio } & 1 & $-0,6992 *$ & $-0,1267^{\mathrm{ns}}$ & $0,4408^{*}$ & $-0,7553^{*}$ & $-0,0371^{\mathrm{ns}}$ & $0,2370^{\mathrm{ns}}$ \\
\hline & 2 & $-0,6014^{*}$ & $-0,2712^{\mathrm{ns}}$ & $0,3941 *$ & $-0,8049^{*}$ & $0,0290^{\mathrm{ns}}$ & $0,2992^{\mathrm{ns}}$ \\
\hline & 3 & $-0,6130^{*}$ & $-0,2657^{\mathrm{ns}}$ & $0,3780^{*}$ & $-0,7685^{*}$ & $-0,0022^{\mathrm{ns}}$ & $0,2844^{\mathrm{ns}}$ \\
\hline \multirow{3}{*}{ junho } & 1 & $-0,5054^{*}$ & $-0,4061 *$ & $0,2478^{\mathrm{ns}}$ & $-0,6690^{*}$ & $-0,1730^{\mathrm{ns}}$ & $0,1880^{\mathrm{ns}}$ \\
\hline & 2 & $-0,5488^{*}$ & $-0,3736^{*}$ & $0,2008^{\mathrm{ns}}$ & $-0,7006^{*}$ & $-0,1197^{\mathrm{ns}}$ & $0,1232^{\mathrm{ns}}$ \\
\hline & 3 & $-0,3231 *$ & $-0,5335^{*}$ & $0,1830^{\mathrm{ns}}$ & $-0,5871^{*}$ & $-0,2535^{\mathrm{ns}}$ & $0,2314^{\mathrm{ns}}$ \\
\hline \multirow{3}{*}{ julho } & 1 & $-0,3700^{*}$ & $-0,5187^{*}$ & $0,2266^{\mathrm{ns}}$ & $-0,6181^{*}$ & $-0,2178^{\mathrm{ns}}$ & $0,2397^{\mathrm{ns}}$ \\
\hline & 2 & $-0,3392 *$ & $-0,5037^{*}$ & $0,2643^{\mathrm{ns}}$ & $-0,6656^{*}$ & $-0,1464^{\mathrm{ns}}$ & $0,3553 *$ \\
\hline & 3 & $-0,2213^{\mathrm{ns}}$ & $-0,6223^{*}$ & $0,3179^{*}$ & $-0,5615^{*}$ & $-0,2835^{\mathrm{ns}}$ & $0,3042^{\mathrm{ns}}$ \\
\hline \multirow{3}{*}{ agosto } & 1 & $-0,2505^{\mathrm{ns}}$ & $-0,6084 *$ & $0,2923^{\mathrm{ns}}$ & $-0,5685^{*}$ & $-0,2670^{\mathrm{ns}}$ & $0,3149^{*}$ \\
\hline & 2 & $-0,1683^{\mathrm{ns}}$ & $-0,5963^{*}$ & $0,3754 *$ & $-0,4872 *$ & $-0,3781^{*}$ & $0,4544^{*}$ \\
\hline & 3 & $-0,2205^{\mathrm{ns}}$ & $-0,6103 *$ & $0,3835^{*}$ & $-0,4200^{*}$ & $-0,4852 *$ & $0,3323^{*}$ \\
\hline \multirow{3}{*}{ setembro } & 1 & $-0,1159^{\mathrm{ns}}$ & $-0,6569 *$ & $0,4030^{*}$ & $-0,3888^{*}$ & $-0,4739 *$ & $0,3826^{*}$ \\
\hline & 2 & $-0,1864^{\mathrm{ns}}$ & $-0,6058^{*}$ & $0,3865^{*}$ & $-0,4950^{*}$ & $-0,4250^{*}$ & $0,3248^{*}$ \\
\hline & 3 & $-0,2457^{\mathrm{ns}}$ & $-0,5349^{*}$ & $0,4956^{*}$ & $-0,5879^{*}$ & $-0,2959^{\mathrm{ns}}$ & $0,4817^{*}$ \\
\hline \multirow{3}{*}{ outubro } & 1 & $-0,2440^{\mathrm{ns}}$ & $-0,5628^{*}$ & $0,4488^{*}$ & $-0,5766^{*}$ & $-0,2932^{\mathrm{ns}}$ & $0,4828^{*}$ \\
\hline & 2 & $-0,3513^{*}$ & $-0,4757^{*}$ & $0,5177^{*}$ & $-0,6274^{*}$ & $-0,2461^{\mathrm{ns}}$ & $0,5006^{*}$ \\
\hline & 3 & $-0,4117^{*}$ & $-0,4025^{*}$ & $0,5455^{*}$ & $-0,6286^{*}$ & $-0,2401^{\mathrm{ns}}$ & $0,5465^{*}$ \\
\hline \multirow{3}{*}{ novembro } & 1 & $-0,3757^{*}$ & $-0,4245^{*}$ & $0,5370^{*}$ & $-0,6580^{*}$ & $-0,1892^{\mathrm{ns}}$ & $0,5835^{*}$ \\
\hline & 2 & $-0,2884^{\mathrm{ns}}$ & $-0,4883^{*}$ & $0,5901^{*}$ & $-0,6001^{*}$ & $-0,2335^{\mathrm{ns}}$ & $0,6027^{*}$ \\
\hline & 3 & $-0,3952 *$ & $-0,3471^{*}$ & $0,6381^{*}$ & $-0,6180^{*}$ & $-0,1538^{\mathrm{ns}}$ & $0,6539^{*}$ \\
\hline \multirow{3}{*}{ dezembro } & 1 & $-0,4590^{*}$ & $-0,2761^{\mathrm{ns}}$ & $0,6533^{*}$ & $-0,6510^{*}$ & $-0,1303^{\mathrm{ns}}$ & $0,6551 *$ \\
\hline & 2 & $-0,4475^{*}$ & $-0,2938^{\mathrm{ns}}$ & $0,6656^{*}$ & $-0,6498^{*}$ & $-0,1395^{\mathrm{ns}}$ & $0,6498 *$ \\
\hline & 3 & $-0,4631^{*}$ & $-0,2073^{\mathrm{ns}}$ & $0,6701^{*}$ & $-0,6664 *$ & $-0,0858^{\mathrm{ns}}$ & $0,6712 *$ \\
\hline
\end{tabular}

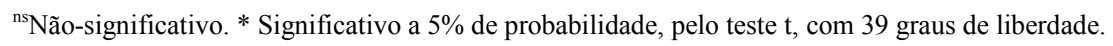

Ciência Rural, v.38, n.9, dez, 2008. 
Coordenadas geográficas na estimativa das temperaturas máxima e média decendiais do ar no Estado do Rio Grande do Sul. 2453

Tabela 3 - Estimativa dos parâmetros $\left(\beta_{0}, \beta_{1}, \beta_{2}\right.$ e $\left.\beta_{3}\right)$ da regressão linear múltipla e do coeficiente de determinação $\left(\mathrm{R}^{2}\right)$, da temperatura máxima média decendial (Tx) em função da altitude $(\mathrm{m})$, da latitude ${ }^{(1)}$ (graus - valores positivos) e da longitude ${ }^{(1)}$ (graus - valores positivos), no Estado do Rio Grande do Sul, com dados de 1945 a 1974.

\begin{tabular}{|c|c|c|c|c|c|c|}
\hline Mês & Decêndio & Constante $\left(\beta_{0}\right)$ & Altitude $\left(\beta_{1}\right)$ & Latitude $\left(\beta_{2}\right)$ & Longitude $\left(\beta_{3}\right)$ & $\mathrm{R}^{2}(\%)$ \\
\hline \multirow{3}{*}{ janeiro } & 1 & 23,91533644 & $-0,00461482$ & $-0,78452182$ & 0,57789845 & 73,71 \\
\hline & 2 & 23,49062331 & $-0,00463639$ & $-0,77563460$ & 0,57710119 & 78,17 \\
\hline & 3 & 21,85572761 & $-0,00478881$ & $-0,74786980$ & 0,59785640 & 77,90 \\
\hline \multirow{3}{*}{ fevereiro } & 1 & 28,10715339 & $-0,00479055$ & $-0,86382332$ & 0,54020629 & 81,18 \\
\hline & 2 & 22,13290830 & $-0,00508432$ & $-0,72070105$ & 0,56289590 & 83,49 \\
\hline & 3 & 24,59104491 & $-0,00499530$ & $-0,76957102$ & 0,54436666 & 81,10 \\
\hline \multirow{3}{*}{ março } & 1 & 33,34476600 & $-0,00512696$ & $-0,86384718$ & 0,42788118 & 79,86 \\
\hline & 2 & 37,51648721 & $-0,00537289$ & $-0,80731538$ & 0,29553359 & 79,61 \\
\hline & 3 & 34,40278836 & $-0,00484727$ & $-0,72714669$ & 0,29491761 & 79,10 \\
\hline \multirow{3}{*}{ abril } & 1 & 39,24030186 & $-0,00542578$ & $-0,87089147$ & 0,25733478 & 85,88 \\
\hline & 2 & 43,31764094 & $-0,00560095$ & $-0,88727432$ & 0,16667941 & 85,46 \\
\hline & 3 & 47,16428640 & $-0,00559880$ & $-0,88549927$ & 0,07234975 & 82,06 \\
\hline \multirow{3}{*}{ maio } & 1 & 36,48913692 & $-0,00493337$ & $-0,69927900$ & 0,14993603 & 83,76 \\
\hline & 2 & 43,15928098 & $-0,00516242$ & $-0,90422753$ & 0,12411813 & 83,61 \\
\hline & 3 & 40,92057720 & $-0,00462011$ & $-0,79710136$ & 0,09121728 & 84,62 \\
\hline \multirow{3}{*}{ junho } & 1 & 50,34203137 & $-0,00478413$ & $-0,97325525$ & $-0,01122903$ & 85,84 \\
\hline & 2 & 48,63816103 & $-0,00456680$ & $-0,87216939$ & $-0,05825999$ & 89,12 \\
\hline & 3 & 53,79735675 & $-0,00425694$ & $-1,08034104$ & $-0,02973947$ & 77,08 \\
\hline \multirow{3}{*}{ julho } & 1 & 49,22546216 & $-0,00416883$ & $-1,01475754$ & $-0,00177322$ & 82,01 \\
\hline & 2 & 48,57788380 & $-0,00413855$ & $-1,04227402$ & 0,04933326 & 74,29 \\
\hline & 3 & 47,84309464 & $-0,00370812$ & $-1,16022002$ & 0,13097096 & 80,35 \\
\hline \multirow{3}{*}{ agosto } & 1 & 51,66712213 & $-0,00415722$ & $-1,23144248$ & 0,10078393 & 81,12 \\
\hline & 2 & 44,73172537 & $-0,00342864$ & $-1,19360921$ & 0,24334561 & 71,11 \\
\hline & 3 & 48,33599969 & $-0,00408311$ & $-1,31113336$ & 0,23413947 & 80,32 \\
\hline \multirow{3}{*}{ setembro } & 1 & 44,00617589 & $-0,00319888$ & $-1,26240474$ & 0,29003955 & 77,89 \\
\hline & 2 & 45,98193847 & $-0,00369653$ & $-1,25542159$ & 0,25211950 & 75,40 \\
\hline & 3 & 38,76471584 & $-0,00376506$ & $-1,19634101$ & 0,38360351 & 76,27 \\
\hline \multirow{3}{*}{ outubro } & 1 & 44,28782126 & $-0,00394740$ & $-1,24112758$ & 0,31527904 & 77,89 \\
\hline & 2 & 40,44166859 & $-0,00457815$ & $-1,22282432$ & 0,38541436 & 81,18 \\
\hline & 3 & 37,68644777 & $-0,00463035$ & $-1,10852213$ & 0,39882244 & 79,76 \\
\hline \multirow{3}{*}{ novembro } & 1 & 39,81197930 & $-0,00473279$ & $-1,19744557$ & 0,42985260 & 77,59 \\
\hline & 2 & 35,86141558 & $-0,00407879$ & $-1,24168764$ & 0,54134843 & 81,05 \\
\hline & 3 & 29,13233537 & $-0,00462474$ & $-1,12212157$ & 0,62718455 & 77,40 \\
\hline \multirow{3}{*}{ dezembro } & 1 & 26,99554879 & $-0,00459889$ & $-0,97010850$ & 0,59364133 & 77,68 \\
\hline & 2 & 26,97950148 & $-0,00445644$ & $-0,97425184$ & 0,60161143 & 79,62 \\
\hline & 3 & 21,41840542 & $-0,00432743$ & $-0,84127018$ & 0,64851459 & 72,09 \\
\hline
\end{tabular}

${ }^{(1)}$ Foram usados valores positivos de latitude e de longitude para obtenção das estimativas dos parâmetros do modelo de regressão linear múltipla. 
Tabela 4 - Estimativa dos parâmetros $\left(\beta_{0}, \beta_{1}, \beta_{2}\right.$ e $\left.\beta_{3}\right)$ da regressão linear múltipla e do coeficiente de determinação $\left(\mathrm{R}^{2}\right)$, da temperatura média decendial $(\mathrm{Tm})$ em função da altitude $(\mathrm{m})$, da latitude ${ }^{(1)}$ (graus - valores positivos) e da longitude ${ }^{(1)}$ (graus - valores positivos), no Estado do Rio Grande do Sul, com dados de 1945 a 1974.

\begin{tabular}{|c|c|c|c|c|c|c|}
\hline Mês & Decêndio & Constante $\left(\beta_{0}\right)$ & Altitude $\left(\beta_{1}\right)$ & Latitude $\left(\beta_{2}\right)$ & Longitude $\left(\beta_{3}\right)$ & $\mathrm{R}^{2}(\%)$ \\
\hline \multirow{3}{*}{ janeiro } & 1 & 19,81266137 & $-0,00478418$ & $-0,59783141$ & 0,42923408 & 89,39 \\
\hline & 2 & 20,89661135 & $-0,00519765$ & $-0,55466841$ & 0,38337790 & 92,69 \\
\hline & 3 & 18,79606717 & $-0,00506472$ & $-0,53617409$ & 0,42042675 & 91,09 \\
\hline \multirow{3}{*}{ fevereiro } & 1 & 26,04416244 & $-0,00518620$ & $-0,61552543$ & 0,32242851 & 91,03 \\
\hline & 2 & 24,84662947 & $-0,00532572$ & $-0,50895738$ & 0,28044970 & 93,06 \\
\hline & 3 & 22,58506251 & $-0,00516934$ & $-0,49364472$ & 0,31090219 & 91,65 \\
\hline \multirow{3}{*}{ março } & 1 & 25,48967015 & $-0,00543598$ & $-0,49507048$ & 0,25172010 & 89,64 \\
\hline & 2 & 33,31753731 & $-0,00548227$ & $-0,56224213$ & 0,12058569 & 91,23 \\
\hline & 3 & 29,29454609 & $-0,00500009$ & $-0,48833163$ & 0,14219128 & 90,87 \\
\hline \multirow{3}{*}{ abril } & 1 & 31,88393252 & $-0,00501292$ & $-0,49815030$ & 0,07022203 & 86,40 \\
\hline & 2 & 35,23836346 & $-0,00527907$ & $-0,55869787$ & 0,01818170 & 87,30 \\
\hline & 3 & 33,58521309 & $-0,00487828$ & $-0,49353605$ & $-0,01409731$ & 88,53 \\
\hline \multirow{3}{*}{ maio } & 1 & 36,30028917 & $-0,00467746$ & $-0,56021995$ & $-0,04366420$ & 81,97 \\
\hline & 2 & 31,37442242 & $-0,00469531$ & $-0,50036083$ & 0,00158174 & 84,92 \\
\hline & 3 & 31,06944769 & $-0,00442665$ & $-0,49982352$ & $-0,00364069$ & 80,52 \\
\hline \multirow{3}{*}{ junho } & 1 & 38,06618852 & $-0,00436718$ & $-0,64387977$ & $-0,07194313$ & 82,05 \\
\hline & 2 & 38,78859159 & $-0,00452724$ & $-0,61333517$ & $-0,12471535$ & 83,28 \\
\hline & 3 & 33,66985938 & $-0,00362119$ & $-0,60970137$ & $-0,02277761$ & 76,52 \\
\hline \multirow{3}{*}{ julho } & 1 & 32,77586091 & $-0,00384532$ & $-0,61418954$ & $-0,02179047$ & 77,31 \\
\hline & 2 & 26,70326910 & $-0,00359016$ & $-0,52337163$ & 0,05583239 & 77,49 \\
\hline & 3 & 31,42921592 & $-0,00355689$ & $-0,63480393$ & 0,03166767 & 76,26 \\
\hline \multirow[t]{3}{*}{ agosto } & 1 & 30,12396975 & $-0,00349315$ & $-0,61181338$ & 0,03905115 & 75,18 \\
\hline & 2 & 28,71050622 & $-0,00341846$ & $-0,72520823$ & 0,15876768 & 82,19 \\
\hline & 3 & 37,97865728 & $-0,00374544$ & $-0,87907736$ & 0,07378669 & 85,39 \\
\hline \multirow[t]{3}{*}{ setembro } & 1 & 35,28312905 & $-0,00362692$ & $-0,88441531$ & 0,13362630 & 79,51 \\
\hline & 2 & 40,62468839 & $-0,00424220$ & $-0,89701694$ & 0,05578275 & 87,74 \\
\hline & 3 & 32,37071103 & $-0,00450073$ & $-0,82866808$ & 0,19473912 & 88,12 \\
\hline \multirow[t]{3}{*}{ outubro } & 1 & 32,29716518 & $-0,00443158$ & $-0,82011324$ & 0,20134012 & 85,71 \\
\hline & 2 & 31,42184684 & $-0,00467287$ & $-0,80114655$ & 0,21121885 & 88,86 \\
\hline & 3 & 30,17221879 & $-0,00483277$ & $-0,82967544$ & 0,27048474 & 90,72 \\
\hline \multirow{3}{*}{ novembro } & 1 & 27,75475574 & $-0,00504261$ & $-0,80770777$ & 0,32222172 & 91,43 \\
\hline & 2 & 26,92028387 & $-0,00467594$ & $-0,82045839$ & 0,35534164 & 88,61 \\
\hline & 3 & 20,80222337 & $-0,00482555$ & $-0,76248882$ & 0,46168140 & 85,86 \\
\hline \multirow{3}{*}{ dezembro } & 1 & 21,57144955 & $-0,00489724$ & $-0,73401664$ & 0,44238884 & 88,59 \\
\hline & 2 & 22,77596472 & $-0,00490790$ & $-0,74575358$ & 0,43199503 & 88,98 \\
\hline & 3 & 19,27965728 & $-0,00480700$ & $-0,66628996$ & 0,46431236 & 87,78 \\
\hline
\end{tabular}

(1) Foram usados valores positivos de latitude e de longitude para obtenção das estimativas dos parâmetros do modelo de regressão linear múltipla.

Ciência Rural, v.38, n.9, dez, 2008. 
na estimativa da $\mathrm{Tx}$ de $0,454240^{\circ} \mathrm{C}$ e na $\mathrm{Tm}$ de $0,459012^{\circ} \mathrm{C}$ a cada acréscimo de $100 \mathrm{~m}$ de altitude. Esses valores são próximos ao gradiente adiabático úmido da troposfera e semelhantes aos resultados de ALFONSI et al. (1974) e CARGNELUTTI FILHO et al. (2006).

Comportamento semelhante da Tx e Tm é evidenciado em relação às estimativas dos parâmetros relacionados à latitude $\left(\beta_{2}\right)$ e longitude $\left(\beta_{3}\right)$, em que, de maneira geral, maiores magnitudes ocorrem nos decêndios iniciais e finais do ano com decréscimo gradativo para os decêndios centrais do ano. Quanto à Tx e Tm, há, respectivamente, diminuição de aproximadamente $0,70 \mathrm{a} 1,31^{\circ} \mathrm{C}$ e de 0,49 a $0,90^{\circ} \mathrm{C}$ a cada grau de aumento na latitude. Já o acréscimo de um grau na longitude altera a $\mathrm{Tx}$ de $-0,06^{\circ} \mathrm{C}$ a $0,65^{\circ} \mathrm{C}$ e a Tm de 0,13 a $0,46^{\circ} \mathrm{C}$.

Em dez municípios do Rio Grande do Sul utilizados para validação dos modelos obtidos, o coeficiente de correlação linear entre a Tx estimada e a Tx observada oscilou entre 0,8673 e 0,9726 nos 36 decêndios do ano, com média de 0,9386 entre os decêndios, e entre a Tm estimada e a Tm observada variou entre 0,9226 e 0,9920 nos 36 decêndios do ano, com média de 0,9746 , comprovando a adequabilidade dos modelos (Tabela 5). CARGNELUTTI FILHO et al. (2006), ao validarem os modelos para estimativa da temperatura mínima média decendial do ar, encontraram valores entre 0,83 e 0,97 nos 36 decêndios do ano, com média de 0,93 entre os decêndios. Portanto, comprovase a superioridade do ajuste dos modelos na seguinte ordem decrescente: Tm, Tx e Tmin (CARGNELUTTI FILHO et al., 2006) e reforça-se a inferência anteriormente realizada em relação ao coeficiente de determinação $\left(\mathrm{R}^{2}\right)$.

A distribuição espacial dos 41 municípios cujos dados foram utilizados para gerar os modelos de estimativa de Tx e Tm representou a amplitude de variação da altitude, latitude e longitude do Estado do Rio Grande do Sul. Portanto, esses modelos podem ser utilizados para estimar a Tx e a Tm de qualquer ponto do Estado do Rio Grande do Sul em função das coordenadas geográficas, em todos os decêndios do ano.

Desse modo, em determinado local que tenha altitude de $50 \mathrm{~m}$, latitude $30^{\circ} 10^{\prime} 30^{\prime}$ "S e longitude $50^{\circ} 48^{\prime} 10^{\prime \prime} \mathrm{W}$, a estimativa da Tx para o primeiro decêndio de janeiro é $29,37^{\circ} \mathrm{C}$ e é obtida pela equação linear múltipla (Tabela 3 ), conforme o exemplo: $\mathrm{Tx}=\beta_{0}$ $+\beta_{1} *$ altitude $(\mathrm{m})+\beta_{2} *$ latitude (graus - valores positivos) $+\beta_{3}^{*}$ longitude (graus - valores positivos), ou seja, $\mathrm{Tx}=23,91533644-0,00461482 * 50-0,78452182$ $* 30,175000+0,57789845 * 50,802778=29,37^{\circ} \mathrm{C}$. Nesse mesmo local, a estimativa da Tm para o primeiro
Tabela 5 - Estimativa dos coeficientes de correlação linear de Pearson, da temperatura máxima média decendial (Tx) e média decendial (Tm) estimada em função da altitude, latitude e longitude, e a $\mathrm{Tx}$ e a $\mathrm{Tm}$ observadas, respectivamente, em dez municípios do Estado do Rio Grande do Sul, com dados de 1975 a 2004.

\begin{tabular}{|c|c|c|c|}
\hline Mês & Decêndio & $\begin{array}{l}\text { Tx estimada } \mathrm{x} \\
\text { Tx observada } \\
\end{array}$ & $\begin{array}{l}\text { Tm estimada } x \\
\text { Tm observada }\end{array}$ \\
\hline \multirow{3}{*}{ janeiro } & 1 & $0,9673 * *$ & $0,9820 * *$ \\
\hline & 2 & $0,9648 * *$ & $0,9885^{* *}$ \\
\hline & 3 & $0,9654 * *$ & $0,9865 * *$ \\
\hline \multirow{3}{*}{ fevereiro } & 1 & $0,9485^{* *}$ & $0,9756^{* *}$ \\
\hline & 2 & $0,9491 * *$ & $0,9797 * *$ \\
\hline & 3 & $0,9397 * *$ & $0,9776 * *$ \\
\hline \multirow{3}{*}{ março } & 1 & $0,8673^{* *}$ & $0,9907 * *$ \\
\hline & 2 & $0,8898 * *$ & $0,9920 * *$ \\
\hline & 3 & $0,8933^{* *}$ & $0,9887 * *$ \\
\hline \multirow{3}{*}{ abril } & 1 & $0,9676^{* *}$ & $0,9799 * *$ \\
\hline & 2 & $0,9602 * *$ & $0,9721 * *$ \\
\hline & 3 & $0,9515^{* *}$ & $0,9578 * *$ \\
\hline \multirow{3}{*}{ maio } & 1 & $0,9482 * *$ & $0,9874 * *$ \\
\hline & 2 & $0,9464 * *$ & $0,9757 * *$ \\
\hline & 3 & $0,9047 * *$ & $0,9812 * *$ \\
\hline \multirow{3}{*}{ junho } & 1 & $0,9465^{* *}$ & $0,9583 * *$ \\
\hline & 2 & $0,9233 * *$ & $0,9475 * *$ \\
\hline & 3 & $0,8986^{* *}$ & $0,9226^{* *}$ \\
\hline \multirow{3}{*}{ julho } & 1 & $0,9580^{* *}$ & $0,9570 * *$ \\
\hline & 2 & $0,9027 * *$ & $0,9775 * *$ \\
\hline & 3 & $0,9386^{* *}$ & $0,9702 * *$ \\
\hline \multirow{3}{*}{ agosto } & 1 & $0,9048 * *$ & $0,9357 * *$ \\
\hline & 2 & $0,8944 * *$ & $0,9589 * *$ \\
\hline & 3 & $0,9564 * *$ & $0,9683 * *$ \\
\hline \multirow{3}{*}{ setembro } & 1 & $0,9192 * *$ & $0,9674 * *$ \\
\hline & 2 & $0,9309 * *$ & $0,9829 * *$ \\
\hline & 3 & $0,9267 * *$ & $0,9861 * *$ \\
\hline \multirow{3}{*}{ outubro } & 1 & $0,9508 * *$ & $0,9778 * *$ \\
\hline & 2 & $0,9653 * *$ & $0,9836 * *$ \\
\hline & 3 & $0,9549^{* *}$ & $0,9889 * *$ \\
\hline \multirow{3}{*}{ novembro } & 1 & $0,9559^{* *}$ & $0,9855^{* *}$ \\
\hline & 2 & $0,9250 * *$ & $0,9741 * *$ \\
\hline & 3 & $0,9704^{* *}$ & $0,9777^{* *}$ \\
\hline \multirow{3}{*}{ dezembro } & 1 & $0,9726^{* *}$ & $0,9855^{* *}$ \\
\hline & 2 & $0,9615 * *$ & $0,9851 * *$ \\
\hline & 3 & $0,9709 * *$ & $0,9804 * *$ \\
\hline
\end{tabular}

${ }^{\text {ns Não-significativo. } * * \text { Significativo a } 1 \% \text { de probabilidade, pelo }}$ teste t, com 8 graus de liberdade. 
decêndio de janeiro é $23,34^{\circ} \mathrm{C}$ e é obtida pela equação linear múltipla (Tabela 4), conforme o exemplo: $\mathrm{Tm}=\beta_{0}$ $+\beta_{1} *$ altitude $(\mathrm{m})+\beta_{2} *$ latitude (graus - valores positivos) $+\beta_{3} *$ longitude (graus - valores positivos), ou seja, $\mathrm{Tm}=19,81266137-0,00478418 * 50-0,59783141$ $* 30,175000+0,42923408 * 50,802778=23,34^{\circ} \mathrm{C}$.

\section{CONCLUSÃO}

A temperatura máxima média decendial e a temperatura média decendial do ar, no Estado do Rio Grande do Sul, em qualquer local e decêndio, pode ser estimada por meio da altitude, latitude e longitude.

\section{AGRADECIMENTOS}

Os autores agradecem aos pesquisadores, técnicos, observadores meteorológicos, estagiários e às demais pessoas que, de alguma forma, contribuíram para a realização deste trabalho. Os autores agradecem também ao $8^{\circ}$ Distrito de Meteorologia do Instituto Nacional de Meteorologia e à Fundação Estadual de Pesquisa Agropecuária pelos dados e ao Conselho Nacional de Desenvolvimento Científico e Tecnológico $(\mathrm{CNPq})$, pela concessão de bolsa de produtividade em pesquisa a Ronaldo Matzenauer.

\section{REFERÊNCIAS}

ALFONSI, R.R. et al. Estimativas das normais de temperaturas média mensal e anual do Estado de Goiás (BR) em função de altitude e latitude. Caderno de Ciências da Terra, v.45, p.16,1974 .

BURIOL, G.A. et al. Estimativa das médias das temperaturas máximas mensais e anuais do Estado do Rio Grande do Sul. Revista do Centro de Ciências Rurais, v.3, p.131-150, 1973.

BURIOL, G.A. et al. Estimativa das médias das temperaturas máximas mensais e anuais do Estado de Santa Catarina. Revista do Centro de Ciências Rurais, v.4, p.81-102, 1974.

CARGNELUTTI FILHO, A. et al. Altitude e coordenadas geográficas na estimativa da temperatura mínima média decendial do ar no estado do Rio Grande do Sul. Pesquisa Agropecuária Brasileira, v.41, p.893-901, 2006.

COELHO, D.T. et al. Estimativa das temperaturas médias mensais e anual no Estado de Minas Gerais. Revista Ceres, v.20, p.455-459, 1973.

CRUZ, C.D. Programa genes: versão Windows; aplicativo computacional em genética e estatística. Viçosa: UFV, 2001. $648 \mathrm{p}$.

DRAPER, N.R.; SMITH, R. Applied regression analysis. 3.ed. New York: Wiley, 1996. 706p.

ESTEFANEL, V. et al. Estimativa das médias das temperaturas mínimas mensais e anuais do Estado do Rio Grande do Sul. Revista do Centro de Ciências Rurais, v.3, p.1-20, 1973.
FEITOZA, L.R. et al. Estimativas das temperaturas médias das máximas mensais e anual do Estado do Espírito Santo. Revista do Centro de Ciências Rurais, v.10, p.25-32, 1980 b.

FEITOZA, L.R. et al. Estimativas das temperaturas médias das mínimas mensais e anual do Estado do Espírito Santo. Revista do Centro de Ciências Rurais, v.10, p.15-24, 1980a.

FERREIRA, M. et al. Estimativa das temperaturas médias mensais e anuais do Estado do Rio Grande do Sul. Revista do Centro de Ciências Rurais, v.1, p.21-52, 1971.

FERREIRA, M. et al. Estimativa das temperaturas médias mensais e anuais do Estado de Santa Catarina. Revista do Centro de Ciências Rurais, v.4, p.19-38, 1974.

HOFFMANN, R.; VIEIRA, S. Análise de regressão: uma introdução à econometria. 3.ed. São Paulo: Hucitec/Edusp, 1998. 379p.

INSTITUTO DE PESQUISAS AGRONÔMICAS. Secção de Ecologia Agrícola. Atlas agroclimático do Estado do Rio Grande do Sul. Porto Alegre, 1989. 3v.

LIMA, M.G.; RIBEIRO, V.Q. Equações de estimativa da temperatura do ar para o Estado do Piauí. Revista Brasileira de Agrometeorologia, v.6, p.221-227, 1998.

MEDEIROS, S.S. et al. Estimativa e espacialização das temperaturas do ar mínimas, médias e máximas na Região Nordeste do Brasil. Revista Brasileira de Engenharia Agrícola e Ambiental, v.9, p.247-255, 2005.

NETER, J. et al. Applied linear regression models. 3.ed. New York: McGraw Hill, 1997. 532p.

OLIVEIRA NETO, S.N. et al. Estimativa de temperaturas mínima, média e máxima do território brasileiro situado entre 16 e $24^{\circ}$ latitude sul e 48 e $60^{\circ}$ longitude oeste. Engenharia na Agricultura, v.10, p.8-17, 2002.

PEDRO JÚNIOR, M.J. et al. Estimativa das temperaturas médias mensais das máximas e das mínimas para o Estado de São Paulo. Campinas: Instituto Agronômico, 1991. 11p. (Boletim Técnico, 142).

PINTO, H.S.; ALFONSI, R.R. Estimativa das temperaturas médias, máximas e mínimas mensais no Estado do Paraná, em função de altitude e latitude. Caderno de Ciências da Terra, v.52, p.1-28, 1974.

PINTO, H.S. et al. Estimativa das temperaturas médias mensais do Estado de São Paulo em função de altitude e latitude. Caderno de Ciências da Terra, v.23, p.1-20, 1972.

SEDIYAMA, G.C.; MELO JÚNIOR, J.C.F. Modelos para estimativa das temperaturas normais mensais médias, máximas, mínimas e anual no Estado de Minas Gerais. Engenharia na Agricultura, v.6, p.57-61, 1998.

SOUZA, G.S. Introdução aos modelos de regressão linear e não-linear. Brasília: Embrapa-SPI/Embrapa-SEA, 1998. $505 \mathrm{p}$.

VALERIANO, M.M.; PICINI, A.G. Uso de sistemas de informação geográfica para a geração de mapas de médias mensais de temperatura do Estado de São Paulo. Revista Brasileira de Agrometeorologia, v.8, p.255-262, 2000. 